\title{
Down-regulation of the global regulator SATB1 by statins in COLO205 colon cancer cells
}

\author{
C.N. LAKSHMINARAYANA REDDY ${ }^{1,5^{*}}$, V.N. VYJAYANTI ${ }^{1 *}$, DIMPLE NOTANI ${ }^{3}$, \\ SANJEEV GALANDE ${ }^{1,4}$ and SRIGIRIDHAR KOTAMRAJU ${ }^{2}$
}

\author{
${ }^{1}$ Discovery Research Division, Institute of Life Sciences, University of Hyderabad Campus, Hyderabad; \\ ${ }^{2}$ Centre for Chemical Biology, Indian Institute of Chemical Technology, Hyderabad; ${ }^{3}$ National Centre for Cell Science, \\ Ganeshkhind, Pune; ${ }^{4}$ Indian Institute of Science Education and Research, Pashan, Pune, India
}

Received May 6, 2010; Accepted July 16, 2010

DOI: $10.3892 / \mathrm{mmr} .2010 .338$

\begin{abstract}
Special AT-rich sequence binding protein 1 (SATB1) regulates the expression of more than 1,000 genes in tumor cells. SATB1 expression has been implicated in metastasis, and its silencing results in reduced cancer progression and the reversion of metastatic cells to normal appearance. Therefore, any compound causing down-regulation of SATB1 expression or activity may be exploited for its therapeutic potential in terms of cancer regression. Earlier studies showed that the 3-hydroxy-3-methylglutaryl coenzymeA (HMG-CoA) reductase inhibitors (statin drugs), which are widely used to treat hypercholesterolemia, possess other pleotropic activities. These are now increasingly gaining attention for their cancer prevention abilities. However, the downstream interplay of the molecular mechanisms of such anti-cancer activities is unclear. Here, we show that SATB1 is down-regulated by statins in a time- and dose-dependent manner in COLO205 cells. This effect was statin-specific as the down-regulation of SATB1 was brought about by hydrophobic statins, such as simvastatin and fluvastatin, but not by hydrophilic pravastatin. Notably, treatment with mevalonate, an intermediate in the cholesterol and isoprenoid biosynthetic pathways, led to the inhibition of SATB1 down-regulation and cytotoxicity mediated by statins. Treatment with the proteasome inhibitors lactacystine and MG-132 inhibited the statin-mediated down-regulation of
\end{abstract}

Correspondence to: Dr Srigiridhar Kotamraju, Centre for Chemical Biology, Indian Institute of Chemical Technology, Uppal Road, Hyderabad 500 607, India

E-mail: giridhar@iict.res.in

Dr Sanjeev Galande, Indian Institute of Science Education and Research, Pashan, Pune 411021, India

E-mail: sanjeev@iiserpune.ac.in

*Contributed equally

${ }^{5}$ Present address: Department of Plant Pathology, College of Sericulture, UAS (B), Chintamani 563125, India

Key words: special AT-rich sequence binding protein 1, statins, isoprenylation, COLO205 cells, cancer
SATB1, suggesting that regulation occurs at the post-translational level. Thus, our results demonstrate a novel molecular mechanism for the anti-cancer activity of statin drugs in colon cancer cells, without invoking significant cytotoxicity.

\section{Introduction}

The 3-hydroxy-3-methylglutaryl coenzymeA (HMG-CoA) reductase inhibitors (statin drugs) are widely used to treat hypercholesterolemia by reducing serum cholesterol levels (1). They act as competitive inhibitors of HMG-CoA reductase, a rate limiting enzyme in the cholesterol and isoprenoid biosynthetic pathways. Emerging research suggests that statins may also have tumoricidal effects and are implicated in tumor regression, although the results have been mixed $(2,3)$. Statins have been shown to regress in vitro and in vivo tumor growth and to inhibit metastasis at clinically relevant doses $(4,5)$. Nevertheless, the intricate molecular mechanism(s) of statininduced cancer cell death are not completely understood.

Special AT-rich sequence binding protein (SATB1) is a matrix attachment region (MAR)-binding protein that participates in higher-order chromatin organization and tissuespecific gene expression. SATB1 has recently been shown to be the 'master regulator' of global gene expression $(6,7)$. Furthermore, phosphorylation of SATB1 acts as a molecular switch in determining whether it acts as a transcriptional activator or repressor (7). At the genome-wide level, SATB1 organizes and regulates transcriptionally poised genes (8). Intriguingly, SATB1 is up-regulated during malignancy, markedly altering the gene expression profile of cancer cells to induce an aggressive phenotype that promotes both tumor growth and metastasis (6). Strikingly, knockdown of SATB1 in aggressive breast cancer cells reverses metastasis (6).

The objective of this study was to examine whether the genome organizer SATB1 is regulated through the cholesterol or isoprenoid biosynthetic pathways. We observed that the inhibition of mevalonate, a common precursor of both the cholesterol and isoprenoid biosynthetic pathways by HMG-CoA reductase inhibitors (statins), or the addition of geranylgeranyl transferase inhibitor (GGTI-298), which specifically and significantly blocks the geranylgeranylation of Rho/Rac GTPases, diminished SATB1 levels in colon cancer COLO205 cells, thereby implicating the isoprenoid-mediated regulation of SATB1. This 
down-regulation of SATB1 protein may in part be responsible for the anti-cancer activities of statin drugs.

\section{Materials and methods}

Reagents. Fluvastatin, simvastatin, geranylgeranyl transferase inhibitor (GGTI)-298 and farnesyl transferase inhibitor (FTI)-277 were from Calbiochem. 3-(4,5-dimethylthiazol2-yl)-2,5 diphenyl tetrazolium bromide (MTT), mevalonate, clasto-lactacystine $\beta$-lactone and MG-132 were from Sigma, Inc. Cell culture media were purchased from Life Technologies.

Cell culture. COLO205, K562 cells were grown in RPMI-1640; HepG2, SK-N-SH cells in DMEM; MCF-7, MDAMB-231 cells in MEM. Medium was supplemented with $10 \%$ FBS, containing penicillin $(100 \mathrm{U} / \mathrm{ml})$, streptomycin $(100 \mu \mathrm{g} / \mathrm{ml})$ and glutamine $(4 \mathrm{mM})$. All cell lines were originally obtained from ATCC.

Cytotoxicity/cell proliferation assay. The effect of statins on the viability of COLO205 cells was monitored by the MTT assay. At $\sim 70 \%$ confluence, cells were treated with simvastatin, fluvastatin or pravastatin at different concentrations $(0-75 \mu \mathrm{M})$ as a function of time in the presence or absence of different concentrations of mevalonate $(0-75 \mu \mathrm{M})$. After the treatments, MTT was added to a final concentration of $1 \mathrm{mg} /$ $\mathrm{ml}$ and further incubated for $1 \mathrm{~h}$ at $37^{\circ} \mathrm{C}$ in a humidified $5 \%$ $\mathrm{CO}_{2}$ incubator. Cells were dissolved in $1 \mathrm{ml}$ of DMSO and readings were taken at $570 \mathrm{~nm}$ in a Spectrophotometer.

Preparation of whole cell extracts. After treatment, cells were homogenized in $200 \mu \mathrm{l}$ RIPA buffer containing protease inhibitor cocktail. The lysate was centrifuged at $750 \mathrm{x} \mathrm{g}$ for $10 \mathrm{~min}$ at $4^{\circ} \mathrm{C}$ to pellet out the nuclei. The remaining supernatant was centrifuged for $30 \mathrm{~min}$ at $13,000 \mathrm{x}$ g. Protein was determined using the Lowry method and $40 \mu \mathrm{g}$ of the lysate was used for Western blot analysis using anti-rabbit SATB1, as previously described (7).

$R T-P C R$. Total RNA was isolated using TRIzol reagent, and cDNA was prepared using Superscript II reverse transcriptase (Invitrogen). Primer sequences used for SATB1 were: forward 5'-GTGGAAGCCTTGGGAATCC-3'; reverse 5'-CTGACAGCTCTTCTTCTAGTT-3'.

\section{Results}

Statin-mediated down-regulation of SATB1. SATB1 is known to be expressed in multiple cell types, but at varying levels (9). Therefore, we screened for the basal levels of SATB1 protein in different carcinoma cell lines. Of the eight different cell lines tested, SATB1 protein levels were found to be highest in COLO205 (colon carcinoma) followed by K562 (erythromyeloblastoid leukaemia), and relatively low levels were observed in SK-N-SH (neuroblastoma) and MCF7 cells (Fig. 1A). Since the endogenous expression of SATB1 was found to be highest in COLO205 cells, we performed subsequent experiments using these cells. To study the effect of HMG-CoA reductase inhibitors on SATB1 levels, COLO205 cells were treated with simvastatin, fluvastatin and pravastatin $(25 \mu \mathrm{M})$ for $48 \mathrm{~h}$. Simvastatin and fluvastatin are hydrophobic, whereas pravastatin is hydrophilic. It was observed that simvastatin and fluvastatin significantly depleted SATB1 protein levels (Fig. 1B), whereas pravastatin had no effect (Fig. 1B), thereby suggesting that only hydrophobic statins are more effective in down-regulating SATB1 levels. To determine whether statinmediated effects on SATB1 protein were due to HMG-CoA reductase inhibition, cells were pre-treated with mevalonate $(50 \mu \mathrm{M})$ prior to the addition of statins. It was found that mevalonate completely reversed simvastatin and fluvastatin induced the down-regulation of SATB1 (Fig. 1B). Next, we studied the dose- and time-dependent effects of statins on SATB1. For this, cells were treated with two different doses (25 and $50 \mu \mathrm{M}$ ) of simvastatin for various time periods $(0-48 \mathrm{~h})$. SATB1 levels appeared to decrease by $24 \mathrm{~h}$ with $50 \mu \mathrm{M}$, and by $36 \mathrm{~h}$ with $25 \mu \mathrm{M}$ of simvastatin (Fig. 1C), and the addition of mevalonate alleviated the down-regulation of SATB1 by simvastatin (Fig. 1C).

Statin-induced cytotoxicity in COLO205: Effect of mevalonate supplementation. We examined the effectiveness of simvastatin, fluvastatin and pravastatin in causing cytotoxicity to COLO205 cells employing the MTT assay. The viability of simvastatin and fluvastatin $(25 \mu \mathrm{M})$-treated cells was reduced to $\sim 75 \%$ compared to untreated conditions by $48 \mathrm{~h}$, whereas in the presence of mevalonate $(50 \mu \mathrm{M})$, cell viability was completely restored. By contrast, pravastatin had no effect on cell viability (Fig. 2A). Next, the cytotoxicity assay was performed in cells treated with various concentrations of simvastatin $(0-75 \mu \mathrm{M})$ in the presence or absence of mevalonate $(50 \mu \mathrm{M})$ for different time periods $(0-48 \mathrm{~h})$. Simvastatin dose- and time-dependently decreased cell viability by $\sim 50 \%$ in COLO205 cells, with maximum effect at 50 and $75 \mu \mathrm{M}$ (Fig. 2B). Similarly, the addition of various concentrations of mevalonate (50-100 $\mu \mathrm{M})$ along with simvastatin $(50 \mu \mathrm{M})$ either completely (at 50 and $75 \mu \mathrm{M}$ mevalonate) or partially (at $25 \mu \mathrm{M}$ mevalonate) protected cells from statininduced cytotoxicity (Fig. 2C).

Down-regulation of SATBI by simvastatin is at the posttranslational level: Effect of proteasomal inhibitors. Having observed that simvastatin down-regulates SATB1 protein in COLO205 cells, we examined whether the regulation of SATB1 by statins occurs at the transcription level. To our surprise, SATB1 transcript levels did not change significantly with statins (Fig. 3A). Owing to this discrepancy, we then proceeded to test whether the proteasomal pathway plays any role in the proteolytic degradation of SATB1 protein in COLO205 cells by statins. To this end, cells were incubated with proteasomal inhibitors, lactacystine $(10 \mu \mathrm{M})$ and MG-132 $(5 \mu \mathrm{M}) 2 \mathrm{~h}$ prior to the addition of simvastatin $(25 \mu \mathrm{M})$ for $48 \mathrm{~h}$. It was found that both the proteasomal inhibitors significantly restored the SATB1 levels as compared to statin alone-treated cells (Fig. 3B), thus implicating the proteasomal enzyme system in the regulation of SATB1 under these experimental conditions. However, the proteasomal inhibitors did not affect statin-induced cytotoxicity (Fig. 3C). Additionally, we investigated the role of isoprenylation in the statin-mediated down-regulation of SATB1 levels. It was observed that the incubation of COLO205 cells with GGTI-298 $(20 \mu \mathrm{M})$, but not with FTI-277 $(20 \mu \mathrm{M})$, significantly reduced SATB1 levels when incubated for $48 \mathrm{~h}$ (Fig. 3D). This 
A

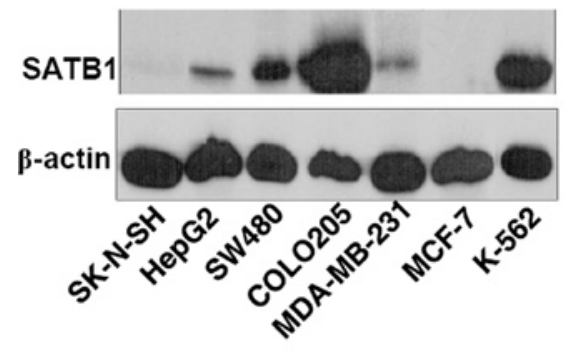

B

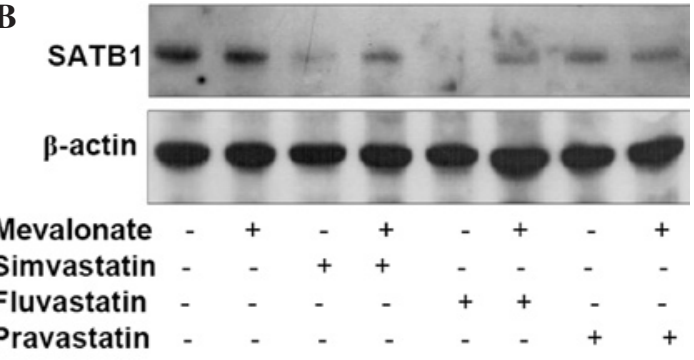

Pravastatin

C
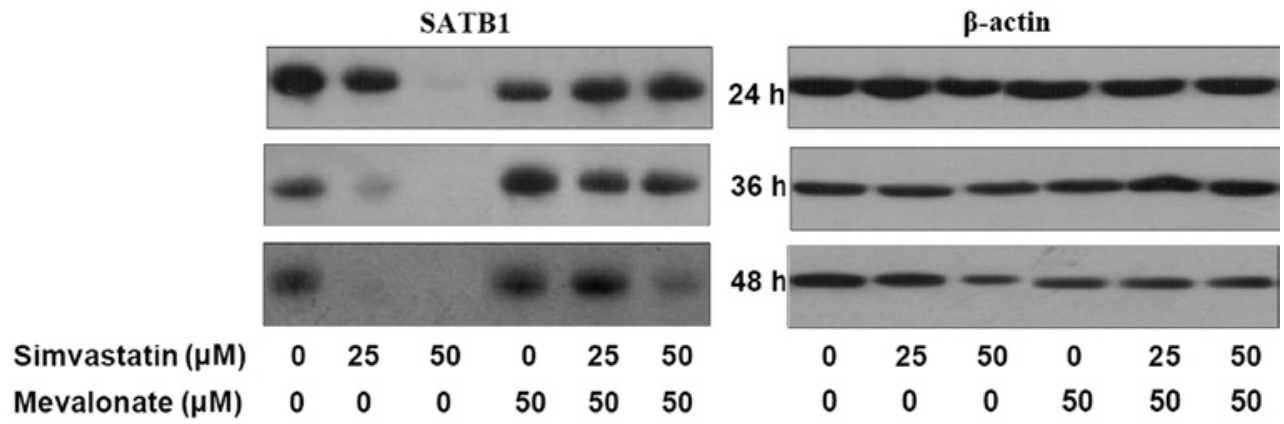

Figure 1. Effect of statins and mevalonate on SATB1 levels in COLO205 cells. (A) Endogenous expression levels of SATB1 protein in various cancerous cell lines. Protein $(40 \mu \mathrm{g})$ from each cell extract was loaded for Western blot analysis. (B) Effect of simvastatin, fluvastatin and pravastatin (at $25 \mu \mathrm{M})$ on SATB1 protein levels, with or without mevalonate $(50 \mu \mathrm{M})$ supplementation in COLO205 cells treated for $48 \mathrm{~h}$. Protein $(40 \mu \mathrm{g})$ from each treatment was used for Western blot analysis. (C) Western blot showing the time- and dose-dependent down-regulation of SATB1 by simvastatin and its reversion with mavelonate supplementation. Panels represent treatments durations of 24,36 and $48 \mathrm{~h}$, respectively. Immunoblot analysis using anti- $\beta$-actin served as the loading control.

A

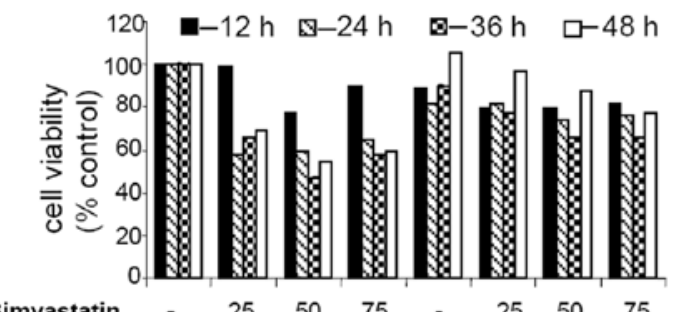

Simvastatin

$(\mu \mathrm{M})$

Mevalonate

$(\mu \mathrm{M})$

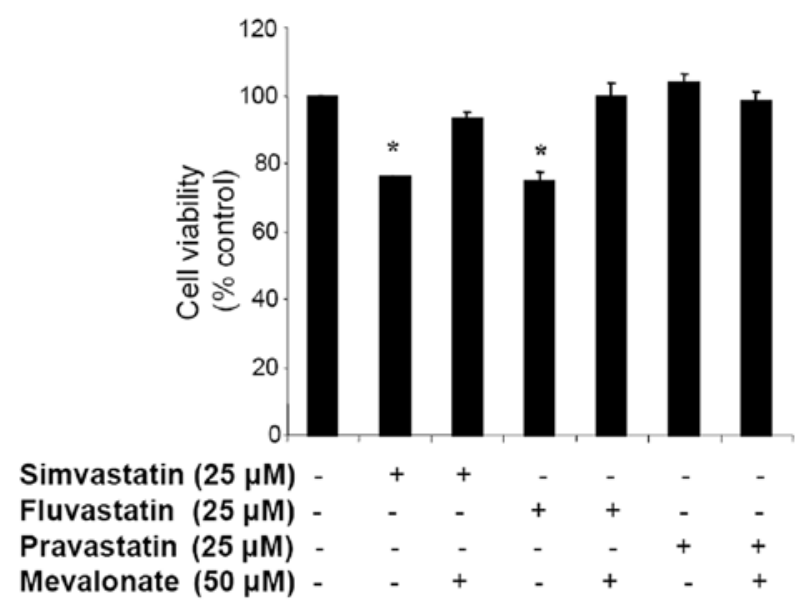

C

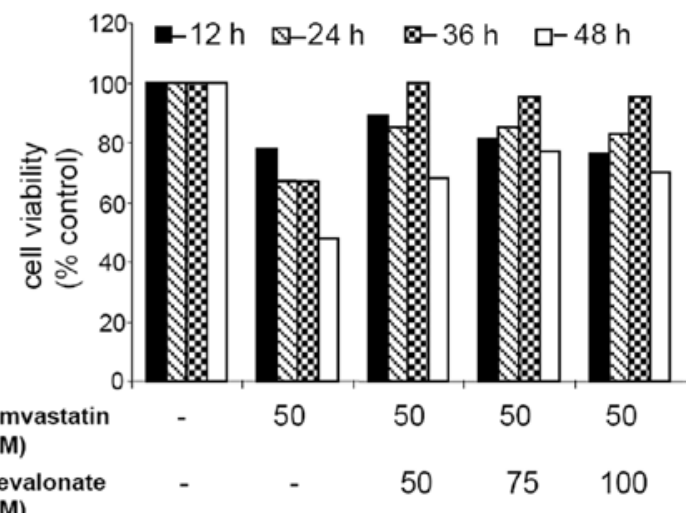

Malonate

$(\mu \mathrm{M})$

Figure 2. Effect of mevalonate on statin-induced cytotoxicity in COLO205 cells. (A) Cells were treated with either simvastatin, fluvastatin or pravastatin $(25 \mu \mathrm{M})$ in the presence or absence of mevalonate $(50 \mu \mathrm{M})$ for $48 \mathrm{~h}$, and cell viability was measured by the MTT assay. (B) Cells were treated with varying concentrations of simvastatin $(25-75 \mu \mathrm{M})$ for different time periods $(12-48 \mathrm{~h})$ in the presence or absence of mevalonate (50 $\mu \mathrm{M})$, and cell death was measured by the MTT assay. (C) As above, except that simvastatin $(50 \mu \mathrm{M})$-induced cell death was measured in the presence of varying concentrations of mevalonate $(50-100 \mu \mathrm{M})$. Values are the mean $\pm \mathrm{SE}$ of three independent experiments. *Significantly different compared to untreated conditions. 
A

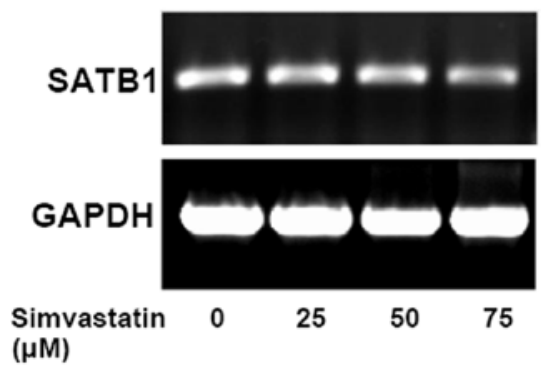

B
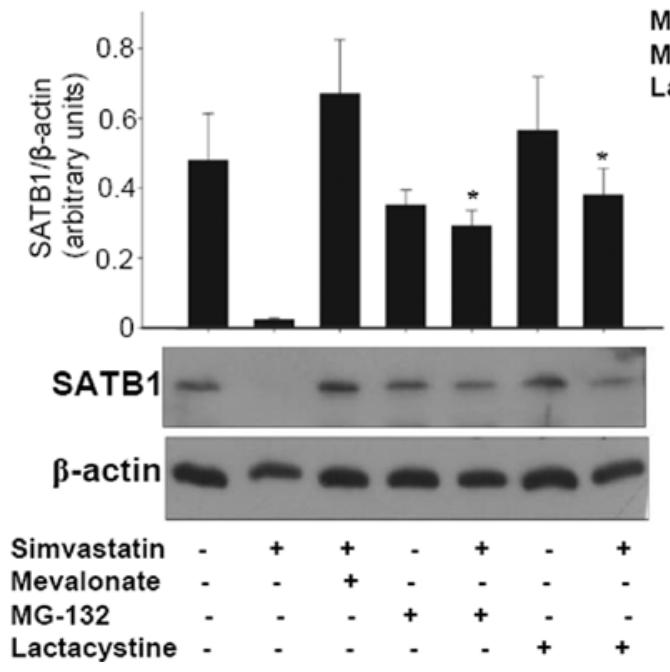

C

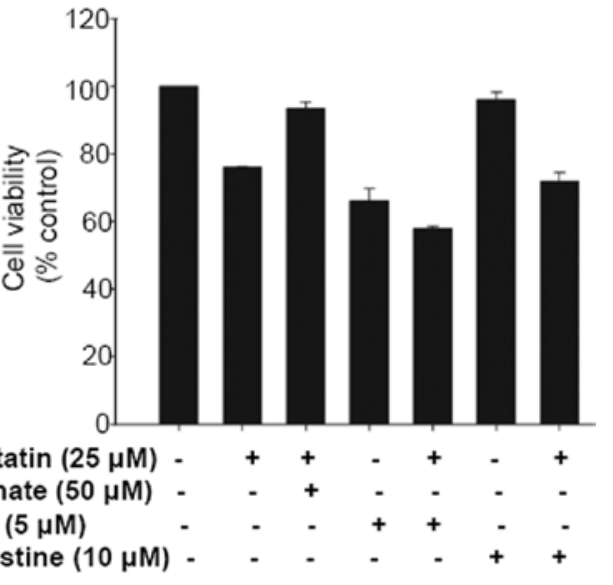

D

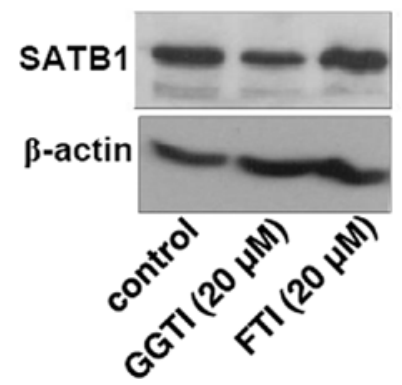

Figure 3. Effect of proteasomal inhibitors and the role of isoprenylation on statin-induced down-regulation of SATB1 in COLO205 cells. (A) SATB1 transcript levels in cells treated with simvastatin at the indicated concentrations was performed by RT-PCR, as described in Materials and methods. GAPDH transcripts served as loading control. (B) Cells were treated with simvastatin $(25 \mu \mathrm{M})$ for $48 \mathrm{~h}$ in the presence or absence of either mevalonate $(50 \mu \mathrm{M})$ or proteasomal inhibitors (MG-132, $5 \mu \mathrm{M}$; lactacystin, $10 \mu \mathrm{M}$ ). Proteasomal inhibitors were added $2 \mathrm{~h}$ prior to the addition of simvastatin. Bar graph depicts normalized band intensity values of SATB1 protein levels of the Western blot panels shown below. (C) Cell viability was estimated using the MTT assay at 48 h. Data are the mean \pm SE of three independent experiments. (D) Cells were treated with either GGTI-298 (20 $\mu \mathrm{M})$ or FTI-277 (20 $\mu \mathrm{M})$ for 48 h, and immunoblotting was performed as described in Materials and methods. *Significantly different from simvastatin alone-treated conditions.

result emphasizes the involvement of the geranylgeranylation of certain Rho or Rac GTPases in regulating SATB1 protein.

\section{Discussion}

Although recent research has shown that SATB1 promotes increased metastasis-associated gene transcriptions on the one hand, and inhibits tumor suppressor genes on the other (6), combined with the earlier reports on the role of SATB1 in the development of thymocytes $(10,11)$, it remains unclear how SATB1 itself is regulated to re-program global gene expression profiles. In this study, we showed that HMG-CoA reductase inhibitors (statins) down-regulate SATB1, thereby implicating either cholesterol intermediates or isoprenoids in the regulation of SATB1 levels in colon carcinoma cells. Moreover, only hydrophobic statins (simvastatin and fluvastatin) and not hydrophilic statins, like pravastatin, were found to be effective in causing altered regulation of SATB1 and increased cytotoxicity. This result is in agreement with recent reports on the differential activity of statins in breast and other gynecological cancers $(5,12)$.

Statins dysregulate the cholesterol and isoprenoid biosynthetic pathways (13). The end-products of these pathways are required for a number of essential cellular functions, including cell motility, proliferation, apoptosis and membrane integrity. Furthermore, they are involved in carcinogenesis (14). Many cancer cell types express elevated levels of HMG-CoA reductase activity to satisfy their needs for increased proliferation, thus making it an attractive chemotherapeutic target (15). Statins have been shown to induce apoptosis, and inhibit cell proliferation in various tumor-derived cells, including colon cancer (16). In a recent population-based case-control study, the use of statins was associated with a $50 \%$ reduction in the risk of colorectal cancer incidence (17).

One of the interesting findings of our study is that, although SATB1 protein is altered by statins, the regulation is not at the transcriptional level. Simvastatin and fluvastatin significantly down-regulated SATB1 protein levels both in a dose- and timedependent manner. We therefore investigated the involvement of the proteasome-mediated degradation of SATB1 protein by these statins. Proteasomal inhibitors significantly restored the SATB1 protein levels to near untreated conditions, implicating that statins may induce post-translational modification(s) of SATB1, which in turn targets it for proteolytic degradation through the proteasomal enzyme machinery. Nevertheless, a thorough understanding of this mechanism must be reached in the near future, along with the regulation of SATB1 by statins in other cancer cell types. 
The data presented here additionally suggest that the cholesterol-independent pathways are responsible for statinmediated STAB1 down-regulation. HMG-CoA reductase inhibitors deplete the availability of prenylated substrates (18). The post-translational prenylation of GTPases, by the addition of geranylgeranyl or farnesyl moiety, is critical for cellular localization and signalling activities (19). In this study, we found that treatment with geranylgeranyl transferase inhibitor (GGTI-298), but not farnesyl transferase inhibitor (FTI-277), resulted in the down-regulation of SATB1, thus mimicking the statin-mediated effects. Therefore, it is likely that statinmediated down-regulation of SATB1 occurs through reduced geranylgeranylation of downstream signaling targets, such as Rho or Rac GTPases.

In conclusion, this is the first report demonstrating that SATB1, the global chromatin organizer and gene regulator, is itself regulated by HMG-CoA reductase inhibitors (statins). This is presumably mediated via the inhibition of the isoprenylation of small GTPases in colon carcinoma cells. However, the detailed mechanism of how this is brought about remains to be further investigated.

\section{Acknowledgements}

The authors thank Ranveer Jayani for help with the preparation of figures and Rafeik Mir for help with the RT-PCR analyses. The study was supported by grants from the DBT, India, and the Wellcome Trust, UK. D.N. is supported by a fellowship from the CSIR, India. S.G. is an international senior research fellow of the Wellcome Trust, UK. The Ramanujan Fellowship to S.K. from the DST, India, is greatly acknowledged.

\section{References}

1. Hoeg JM and Brewer HB Jr: 3-hydroxy-3-methylglutaryl-coenzyme A reductase inhibitors in the treatment of hypercholesterolemia. JAMA 258: 3532-3536, 1987.

2. Katz MS: Therapy insight: potential of statins for cancer chemoprevention and therapy. Nat Clin Pract Oncol 2: 82-89, 2005.

3. Bonovas S, Filioussi K, Tsavaris N and Sitaras NM: Use of statins and breast cancer: a meta-analysis of seven randomized clinical trials and nine observational studies. J Clin Oncol 23 8606-8612, 2005.
4. Matar P, Rozados VR, Roggero EA and Scharovsky OG: Lovastatin inhibits tumor growth and metastasis development of a rat fibrosarcoma. Cancer Biother Radiopharm 13: 387-393, 1998.

5. Kotamraju S, Williams C and Kalyanaraman B: Statin-induced breast cancer cell death: role of inducible nitric oxide and arginine metabolizing enzymes. Cancer Res 67: 7386-7394, 2007.

6. Han HJ, Russo J, Kohwi Y and Kohwi-Shigematsu T: SATB1 reprogrammes gene expression to promote breast tumour growth and metastasis. Nature 452: 187-193, 2008.

7. Kumar PP, Purbey PK, Sinha CK, Notani D, Limaye A, Jayani RS and Galande S: Phosphorylation of SATB1, a global gene regulator, acts as a molecular switch regulating its transcriptional activity in vivo. Mol Cell 22: 231-243, 2006.

8. Galande S, Purbey PK, Notani D and Kumar PP: The third dimension of gene regulation: organization of dynamic chromatin loopscape by SATB1. Curr Opin Genet Dev 17: 408-414, 2007.

9. Kumar PP, Bischof O, Purbey PK, Notani D, Urlaub H, Dejean A and Galande S: Functional interaction between PML and SATB1 regulates chromatin-loop architecture and transcription of the MHC class I locus. Nat Cell Biol 9: 45-56, 2007.

10. Krangel MS: T cell development: better living through chromatin. Nat Immunol 8: 687-694, 2007.

11. Kumar PP, Purbey PK, Ravi DS, Mitra D and Galande S: Displacement of SATB1-bound histone deacetylase 1 corepressor by the human immunodeficiency virus type 1 transactivator induces expression of interleukin-2 and its receptor in T cells. Mol Cell Biol 25: 1620-1633, 2005.

12. Kato S, Smalley S, Sadarangani A, et al: Lipophilic but not hydrophilic statins selectively induce cell death in gynecological cancers expressing high levels of HMGCoA reductase. J Cell Mol Med 14: 1180-1193, 2009.

13. Jakobisiak M and Golab J: Potential antitumor effects of statins. Int J Oncol 23: 1055-1069, 2003.

14. Liao JK: Isoprenoids as mediators of the biological effects of statins. J Clin Invest 110: 285-288, 2002.

15. Duncan RE, EI-Sohemy A and Archer MC: Mevalonate promotes the growth of tumors derived from human cancer cells in vivo and stimulates proliferation in vitro with enhanced cyclin-dependent kinase-2 activity. J Biol Chem 279: 33079-3384, 2004.

16. Agarwal B, Bhendwal S, Halmos B, Moss SF, Ramey WG and Holt PR: Lovastatin augments apoptosis induced by chemotherapeutic agents in colon cancer cells. Clin Cancer Res 5: 2223-2229, 1999.

17. Poynter JN, Gruber SB, Higgins PD, et al: Statins and the risk of colorectal cancer. N Engl J Med 352: 2184-2192, 2005.

18. Schafer WR, Kim R, Sterne R, Thorner J, Kim SH and Rine J: Genetic and pharmacological suppression of oncogenic mutations in ras genes of yeast and humans. Science 245: 379-385, 1989.

19. Kaibuchi K, Kuroda S and Amano M: Regulation of the cytoskeleton and cell adhesion by the rho family GTPases in mammalian cells. Annu Rev Biochem 68: 459-486, 1999. 